\title{
Endotracheal Tuberculous Granuloma Formation Following Endobronchial Ultrasound Transbronchial Needle Aspiration
}

\author{
Jeong-Won Lee ${ }^{1}$, Woo-Jin Kim ${ }^{1}$, Chan-Woo Park ${ }^{1}$, Hyun-Wook Kang ${ }^{1}$, Hee-Jung Ban ${ }^{1}$, \\ In-Jae Oh ${ }^{1}$, Yong-Soo Kwon ${ }^{1}$, Kyu-Sik Kim ${ }^{1}$, Yu-Il Kim ${ }^{1}$, Sung-Chul Lim ${ }^{1}$, \\ Young-Chul Kim ${ }^{1}$ and Yoo-Duk Choi ${ }^{2}$
}

\begin{abstract}
Endobronchial ultrasound-transbronchial needle aspiration (EBUS-TBNA) is a useful and safe diagnostic test. We herein report a case of endotracheal granuloma formation that occurred after EBUS-TBNA in a 73year-old woman. The patient was admitted due to coughing and dyspnea after 70 days of antituberculous therapy for mediastinal lymphadenitis. Computed tomography revealed decreases in the size of the lymph nodes with a new mass protruding into the tracheal lumen. The mass originated from the right paratracheal area, which was a previous puncture site. This case suggests that clinicians should pay attention to complications because tuberculosis can produce new granulomas via the sinus tract after EBUS-TBNA.
\end{abstract}

Key words: complication, endobronchial ultrasound-transbronchial needle aspiration, granuloma, tuberculous lymphadenitis

(Intern Med 52: 1207-1210, 2013)

(DOI: 10.2169/internalmedicine.52.9360)

\section{Introduction}

Endobronchial ultrasound-transbronchial needle aspiration (EBUS-TBNA) is a useful procedure for evaluating the structures within or adjacent to the bronchus. EBUS-TBNA exhibits high sensitivity and specificity in the diagnosis of mediastinal lymphadenopathy (1). Because EBUS-TBNA exhibits higher sensitivity, cost-effectiveness and safety than other surgical procedures, such as mediastinoscopy and mediastinotomy, it is more widely used for diagnosing lymphadenopathy, especially cases of lung cancer (2). Sometimes, postprocedure complications of EBUS-TBNA occur, such as pneumomediastinum, mediastinitis, bleeding and transient bacteremia, which are usually not serious. Only rarely are clinically significant complications reported. We herein report a case of a granuloma that became ingrown into the trachea following EBUS-TBNA in a patient with mediastinal tuberculous lymphadenitis.

\section{Case Report}

A 73-year-old woman was admitted to the orthopedic department with a 10-day history of lower back pain. The patient had no history of trauma or acupuncture. The results of testing for anti-human immunodeficiency virus were negative. Lumbar spine magnetic resonance imaging revealed high signal intensity on the body of the 12th thoracic and 4th lumbar spine. To distinguish a bone tumor from spondylitis, the patient underwent whole-body 18-fludeoxyglucose positron emission tomography $\left({ }^{18} \mathrm{~F}-\mathrm{FDG}\right.$ PET). There were multiple hypermetabolic lymph nodes in the right paratracheal, anterior mediastinal, subcarinal, suprapancreatic and both hilar areas. An 0.8-cm-sized hypermetabolic nodule in the right upper lobe in addition to multiple areas of spine and pelvic bone hypermetabolism were also observed

${ }^{1}$ Department of Internal Medicine, Chonnam National University Medical School, Korea and ${ }^{2}$ Department of Pathology, Chonnam National University Medical School, Korea

Received for publication November 19, 2012; Accepted for publication February 7, 2013

Correspondence to Dr. In-Jae Oh, droij@chonnam.ac.kr 
(Fig. 1a). To make a pathologic diagnosis, EBUS-TBNA (Fig. 2) with a 22-gauge needle for a right paratracheal lymph node and bone biopsy of the 4th lumbar spine were performed under local anesthesia. We attempted to perform TBNA puncture three times, which is usual number of times in our center. When obtaining tissue core specimens, we do not attempt puncture one lymph node more than three times. There were no complications associated with the procedures. A biopsy of the right paratracheal lymph node showed extensive necrosis with focal granuloma formation. The bone biopsy disclosed interstitial fibrosis and chronic inflammation that was compatible with a diagnosis of chronic osteo-

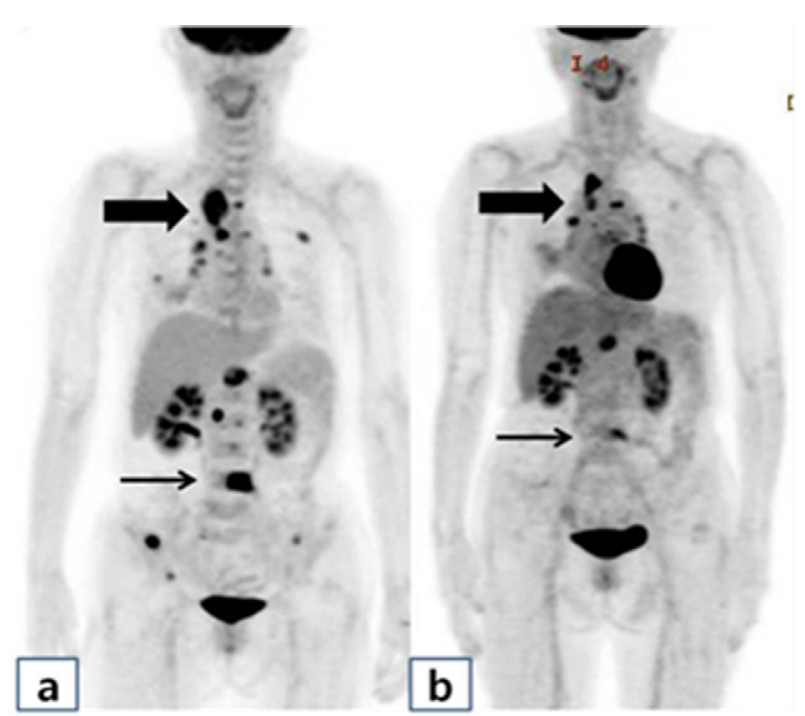

Figure 1. ${ }^{18}$ F-FDG PET imaging showing hypermetabolic lesions: an 0.8-cm-sized lung nodule, a hilar lymph node, a right paratracheal node, a suprapancreatic node, a left 5th rib lesion, a 4th lumbar spine lesion and a pelvic bone lesion. EBUSTBNA of the right paratracheal lymph node (thick arrow) and a bone biopsy of the 4th lumbar spine (thin arrow) were performed (a). After 140 days of antituberculous therapy, significant regression of the hypermetabolic lesions was observed. The right paratracheal lymph node (thick arrow) and 4th lumbar spine (thin arrow) lesions were markedly decreased. myelitis; however, there were no features of tuberculous granuloma (Fig. 3). Mycobacterium tuberculosis was cultured in the bronchoalveolar lavage fluid of the right middle lobe. We initiated antituberculous therapy with isoniazid (300 mg/day), rifampin (450 mg/day) and ethambutol (800 $\mathrm{mg} /$ day) to treat extrapulmonary tuberculosis. The patient did not want to take pyrazinamide due to concerns about gastrointestinal intolerance.

After 70 days of antituberculous therapy, the patient was admitted with coughing and dyspnea. Chest computed tomography revealed a slight decrease in the size of the lymph nodes in addition to a newly detected mass protruding into the tracheal lumen (Fig. 4b). The mass originated from the right paratracheal lymph node area in which the EBUSTBNA had been performed (Fig. 4c). The tracheal mass was removed via rigid bronchoscopy and argon plasma coagulation (APC), and the patient's symptoms immediately disappeared (Fig. 4d). The pathologic findings of the mass were also compatible with a diagnosis of tuberculous granuloma. Because all of the antituberculous drugs were sensitive and CT showed a decrease in the size of the lymph nodes, the patient continued to take these medications.

After 140 days of antituberculous therapy, the patient underwent follow-up ${ }^{18}$ F-FDG PET (Fig. 1b). The multiple areas of mediastinal, spine and pelvic hypermetabolism had markedly improved. However, the patient complained of coughing with chest discomfort, and follow-up bronchoscopy showed renewed growth of the endotracheal granuloma, which was smaller than on the initial examination; therefore, we partially removed the mass via APC under flexible bronchoscopy.

The patient's respiratory symptoms were completely relieved after the second bronchoscopic mass was removed, and the antituberculous therapy was continued until the ninth month. Final follow-up chest CT (Fig. 4e) showed complete resolution of the paratracheal lymphadenopathy and endotracheal granuloma.
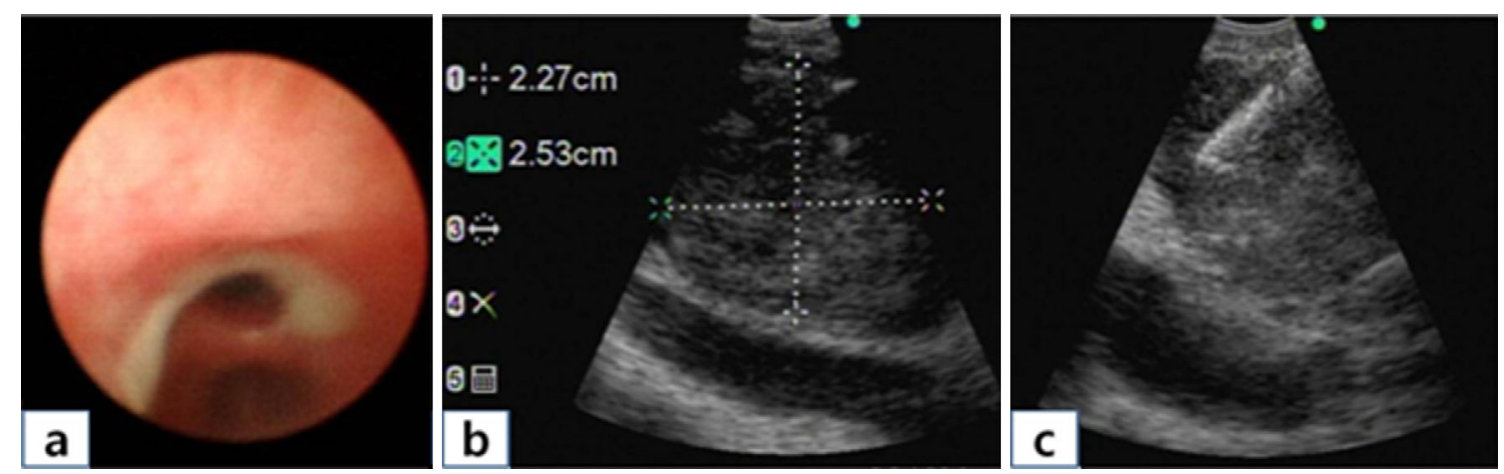

Figure 2. Bronchoscopic findings showing a moderate amount of purulent secretion around the orifice of the right middle lobe (a). Endobronchial ultrasound showing a $2.5-\mathrm{cm}$-sized right paratracheal lymph node (b). The transbronchial needle aspiration procedure was performed on the lymph node (c). 


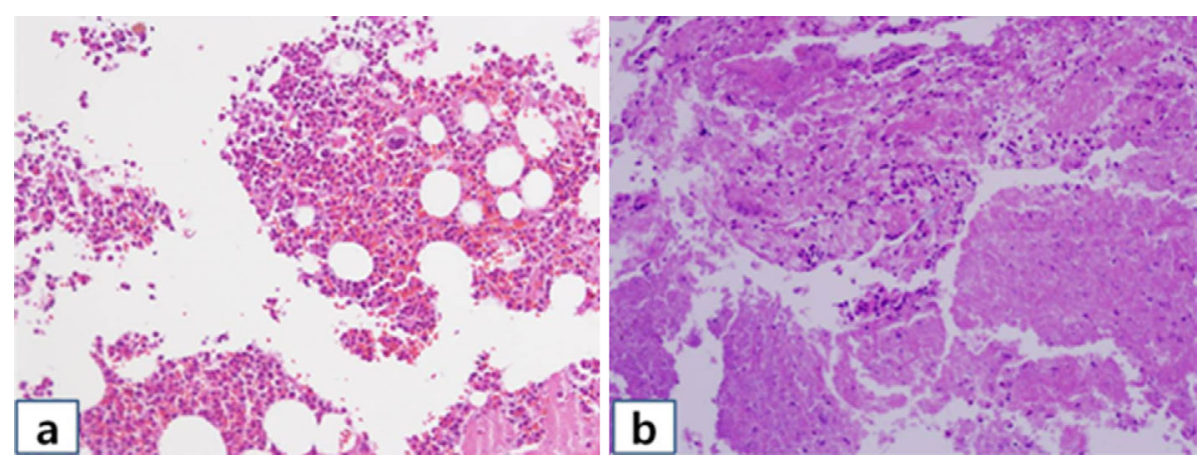

Figure 3. Bone biopsy revealing cellular hematopoietic bone marrow without atypical cells. (a). Pathologic findings of the right paratracheal lymph node showing extensive necrosis with focal granuloma, compatible with a diagnosis of tuberculous lymphadenitis (b). There were no malignant cells in either the bone or lymph node tissue (Hematoxylin and Eosin staining, $\times 100$ ).
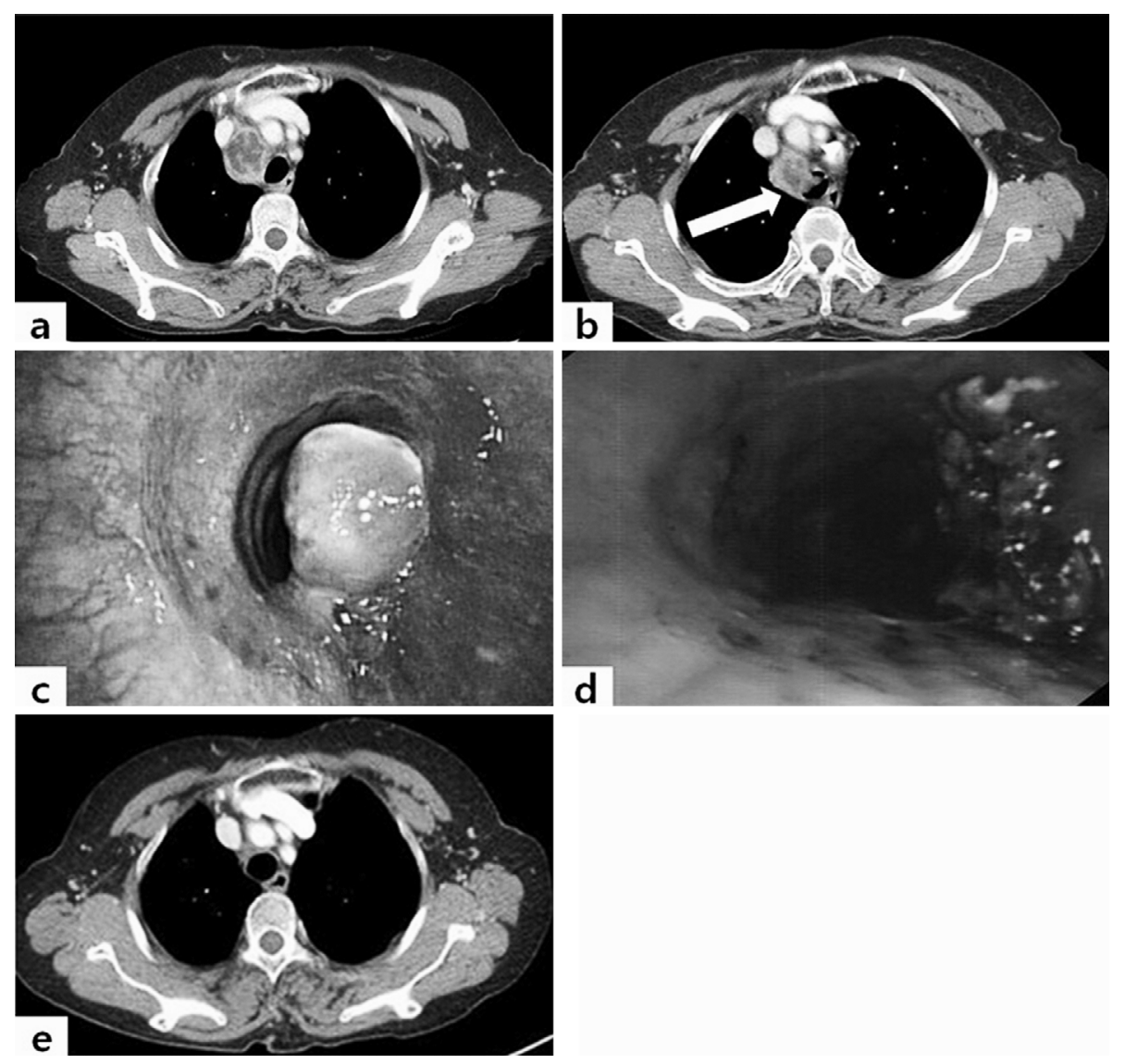

Figure 4. Initial chest CT showing an enlarged necrotizing conglomerated right paratracheal lymph node with peripheral rim enhancement (a). Seventy days after antituberculous therapy, a newly developed granuloma protruding into the tracheal lumen (arrow) was observed despite the fact that the size of the lymph node had somewhat decreased (b). Bronchoscopy showing an endotracheal granuloma on the lateral wall of the lower trachea (c). The lesion was removed and the tracheal lumen was preserved with argon plasma coagulation and rigid bronchoscopy (d). After nine months of antituberculous therapy, final chest CT showed complete resolution of the right paratracheal lymphadenopathy and endotracheal granuloma (e).

\section{Discussion}

To our knowledge, this is the first case of endotracheal granuloma formation following an EBUS-TBNA procedure.
Mediastinal tuberculous lymphadenitis characteristically compresses the major vessels and phrenic or recurrent laryngeal nerves and forms sinuses with the bronchus or esophagus. Reproduction of Mycobacterium tuberculosis in the lymph nodes accompanies delayed hypersensitivity, and in- 
flammation in the center of the lymph node causes lymph node swelling and matting. The center of the lymph node softens, and caseous material can rupture into the surrounding tissue (3). The prevalence of draining sinuses in patients with tuberculous lymphadenitis is estimated to be 2$23 \%$ (4). Most cases of tracheoesophageal fistulas, bronchoesophageal fistulas and esophagomediastinal fistulas are improved with medical treatment only (5-7).

The complications of EBUS-TBNA are generally not serious, and clinically significant complications are rarely reported. Although the needle of EBUS-TBNA is very small (20-22 gauge) and the size of the puncture site is negligible, significant complications can occur. Complications related to the puncture site arise accidentally, such as intramural hematoma and hemopneumomediastinum formation following aortic puncture and cardiac tamponade development following pericardial puncture $(8,9)$. Complications related to the introduction of microorganisms have been reported, including purulent pericarditis, empyema, lung abscesses and mediastinal abscesses $(10,11)$. In this case, we believe that the two-month duration of antituberculous therapy was not adequate because the final CT (Fig. 4e) showed complete resolution after nine months of therapy. In addition, the immune response became more intense after initiating antituberculous therapy. Therefore, the center of the lymph node became soft and caseous material began to swell. Because there was a drainage track in the mediastinal lymph node as a result of EBUS-TBNA, the granuloma was able to grow into the tracheal lumen, although the location of the puncture was accurate and no infection developed.

Because the patient exhibited a radiologic response to antituberculous therapy, it was reasonable to continue the three drugs for a total of nine months for treatment of extrapulmonary tuberculosis (12). If the endotracheal granuloma causes respiratory symptoms again during this period, bronchoscopic removal would be helpful (13). In conclusion, clinicians should be aware of the possibility of granuloma formation along punctured tracts after EBUS-TBNA, especially in patients with mediastinal tuberculous lymphadenitis.

\section{References}

1. Chandra S, Nehra M, Agarwal D, Mohan A. Diagnostic accuracy of endobronchial ultrasound-guided transbronchial needle biopsy in mediastinal lymphadenopathy: a systematic review and metaanalysis. Respir Care 57: 384-391, 2012.

2. Sharples LD, Jackson C, Wheaton E, et al. Clinical effectiveness and cost-effectiveness of endobronchial and endoscopic ultrasound relative to surgical staging in potentially resectable lung cancer: results from the ASTER randomised controlled trial. Health Technol Assess 16: 1-75, iii-iv, 2012.

3. Mohapatra PR, Janmeja AK. Tuberculous lymphadenitis. J Assoc Physicians India 57: 585-590, 2009.

4. Fontanilla JM, Barnes A, von Reyn CF. Current diagnosis and management of peripheral tuberculous lymphadenitis. Clinical Infectious Diseases 53: 555-562, 2011.

5. Lado Lado FL, Golpe Gomez A, Cabarcos Ortiz de Barron A, Antunez Lopez JR. Bronchoesophageal fistulae secondary to tuberculosis. Respiration 69: 362-365, 2002.

6. Ohtake M, Saito H, Okuno M, Yamamoto $S$, Ohgimi $T$. Esophagomediastinal fistula as a complication of tuberculous mediastinal lymphadenitis. Intern Med 35: 984-986, 1996.

7. Lee JH, Shin DH, Kang KW, Park SS, Lee DH. The medical treatment of a tuberculous tracheo-oesophageal fistula. Tuber Lung Dis 73: 177-179, 1992.

8. Gross A, Diacon AH. Cardiac tamponade following transbronchial needle aspiration. Respiration 82: 56-59, 2011.

9. Botana-Rial M, Nunez-Delgado M, Pallares-Sanmartin A, et al. Intramural hematoma of the pulmonary artery and hemopneumomediastinum after endobronchial ultrasound-guided transbronchial needle aspiration. Respiration 83: 353-356, 2012.

10. Haas AR. Infectious complications from full extension endobronchial ultrasound transbronchial needle aspiration. Eur Respir J 33: 935-938, 2009.

11. Huang CT, Chen CY, Ho CC, Yu CJ. A rare constellation of empyema, lung abscess, and mediastinal abscess as a complication of endobronchial ultrasound-guided transbronchial needle aspiration. Eur J Cardiothorac Surg 40: 264-265, 2011.

12. Blumberg HM, Burman WJ, Chaisson RE, et al. American Thoracic Society/Centers for Disease Control and Prevention/Infectious Diseases Society of America: treatment of tuberculosis. Am J Respir Crit Care Med 167: 603-662, 2003.

13. Jin F, Mu D, Xie Y, Fu E, Guo Y. Application of bronchoscopic argon plasma coagulation in the treatment of tumorous endobronchial tuberculosis: Historical controlled trial. J Thorac Cardiovasc Surg 2012 Sep 19, DOI: 10.1016/jjtcvs2012.08.042

The authors state that they have no Conflict of Interest (COI).

(C) 2013 The Japanese Society of Internal Medicine http://www.naika.or.jp/imonline/index.html 